\title{
Comunicação
}

[Communication]

\section{Avaliação pós-operatória da fixação esquelética externa em 29 cães: estudo retrospectivo}

[Post surgical evaluation of external skeletal fixation in 29 dogs: a retrospective study]

\author{
C.M.F. Rezende ${ }^{1}$, H.P. Oliveira ${ }^{1}$, E.M. Penha ${ }^{2}$, E.G. Melo ${ }^{1}$, N.F. Borges ${ }^{2}$, J.V. Doretto ${ }^{2}$ \\ ${ }^{1}$ Escola de Veterinária - UFMG \\ Caixa Postal 567 \\ 303130-970 - Belo Horizonte, MG \\ ${ }^{2}$ Aluno de Pós-Graduação - EV-UFMG
}

A fixação esquelética externa ou transfixação percutânea tem como vantagens a versatilidade, pois pode ser empregada em diversas condições ortopédicas, a invasão tecidual mínima, que possibilita inclusive sua fixação pela técnica fechada, e o baixo custo. É a técnica indicada em fraturas expostas, contaminadas e infectadas e em fraturas com lesão tecidual extensa, como aquela provocada por arma de fogo (Sisk, 1983; Roush, 1992; Carneiro, 2000). Pode ser empregada como meio auxiliar da fixação interna com placas ou pinos. Essa fixação permite o tratamento de feridas na região do foco da fratura sem interferência com o implante e preserva o aporte neurovascular à fratura (Harari et al., 1998; Johnson e DeCamp, 1999). É o método de fixação preconizado para osteotomias corretivas de deformidades do crescimento (Shamir et al., 1996; Harari et al., 1998; Rezende et al., 2000). A remoção do aparelho é rápida e não exige anestesia ou intervenção cirúrgica.

Como outros métodos de fixação de fratura, também a fixação percutânea apresenta complicações que podem interferir na cura óssea (Harari, 1992; Seguin, 1997). Aquelas denominadas complicações maiores são as que podem resultar em falha do procedimento, e as menores são responsáveis pelo desconforto do paciente (Egger, 1991; Gorse, 1998).

A drenagem é a mais comum das complicações menores e pode ter como causa principal o excesso de mobilidade e/ou tensão dos tecidos

Recebido em 16 de junho de 2005

Aceito em 30 de janeiro de 2006

E-mail: cleuza@ufmg.br

Apoio: FAPEMIG/CNPq moles circunjacentes ao pino (Dudley et al., 1997). As complicações maiores são aquelas capazes de levar ao insucesso do tratamento como a incapacidade do aparelho em manter a redução, quebra do pino e não consolidação ou consolidação retardada. Pele e pinos limpos no pós-operatório favorecem o sucesso da intervenção (Egger, 1991, 1998; Harari et al., 1998).

O objetivo deste trabalho foi o estudo retrospectivo das complicações pós-operatórias com o uso de fixação percutânea em cães.

Os dados foram colhidos a partir do arquivo de pacientes do hospital veterinário da Universidade Federal de Minas Gerais de setembro/2000 a julho/2002. Foram separados os casos de acordo com o processo patológico presente, sua localização e tratamento, sendo selecionadas apenas afecções em rádio/ulna e em tíbia/fíbula submetidas à transfixação percutânea.

Foram tratados 29 cães das raças Pinscher (nove, 31,0\%), Pudel (cinco, 17,1\%), Dogue Alemão (dois, 6,9\%) Yorkshire (um, 3,5\%), Fila Brasileiro (um, 3,5\%), Terrier (um, 3,5\%), Greyhound (um, 3,5\%), Dálmata (um, 3,5\%) Schnauzer (um, 3,5\%) e sem raça definida (sete, 24\%).

Foram colhidas informações sobre tipo de curativo empregado e tempo médio de suas trocas, produto utilizado nestes curativos, condição do trajeto dos pinos durante o pósoperatório e no momento da retirada, tempo de permanência da fixação e sucesso/insucesso da terapia. 
Dos 29 casos, dois $(6,9 \%)$ foram submetidos à osteotomia corretiva de rádio curvo, dois $(6,9 \%)$ ao tratamento de fraturas diafisárias simples de tíbia/tíbula, $20(69,0 \%)$ ao tratamento de fraturas diafisárias simples em rádio/ulna e cinco $(17,1 \%)$ casos foram de fratura exposta (um de tíbia/fíbula e quatro de rádio/ulna) e desses, três (60\%) tiveram os aparelhos retirados em 69 dias, em média, com retorno às funções normais, à exceção de um $(20 \%)$ que precisou de tala por maior período devido à fratura de pino e um (20\%) que não retornou para retirada dos pinos (Fig. 1). A configuração de aparelho utilizado foi a do tipo II (bilateral) com barras de conexão em acrílico. A idade média dos animais tratados foi de 18,3 meses, variando dos quatro aos 84 meses, com tempo médio de retirada do aparelho de 66,1 dias, que está dentro do tempo aceitável.

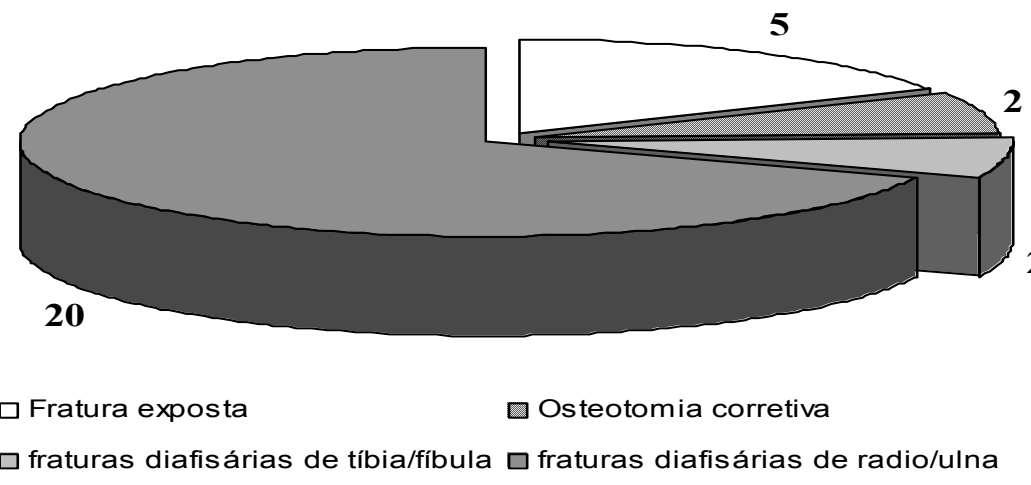

Figura 1. Usos da fixação esquelética externa em 29 cães tratados no hospital veterinário - EV-UFMG.

A secreção ao redor dos pinos e/ou afrouxamento dos implantes foi a complicação de maior casuística $(11,37,9 \%)$ dentre as observadas, de maneira similar ao relatado por Egger (1991), Rudd (1992) e Seguin et al. (1997). Como tratamento pós-operatório foram utilizadas limpeza local com álcool iodado a $0,2 \%$ e repetição da antibióticoterapia (cefalexina, $30 \mathrm{mg} / \mathrm{kg}$, IV TID). O tempo médio de troca das bandagens foi de 8,1 dias, média superior ao preconizado aos proprietários que foi de retorno a cada dois, três, quatro, cinco ou sete dias, dependendo da condição local, tendo a maioria dos casos $(22,75,9 \%)$ indicação de retorno em três ou quatro dias. Esse tempo foi semelhante ao sugerido por Carneiro (2000) para troca da bandagem e limpeza dos pinos. A necessidade desses cuidados pós-operatórios, entretanto, é questão polêmica entre os cirurgiões (Harari, 1992), mesmo sendo a contaminação do trajeto do pino a complicação mais relatada (Harari et al., 1998; Roush, 1992). Geralmente, o cão de raça de pequeno porte e que vive dentro de casa mostra área de perfuração da pele limpa e sem secreção, podendo comparecer para controle semanal.
O tipo de secreção encontrada nos pinos não pôde ser descrito por ausência de informações a este respeito nas fichas médicas, havendo somente informações sobre a sua presença/ausência. Faltaram alguns dados a respeito do peso e idade de alguns animais que foram desconsiderados durante os cálculos.

Foram observados três $(10,4 \%)$ casos de nãounião óssea. Em um deles, o aparelho foi retirado com 249 dias de tratamento, após nova transfixação e enxerto. Esse paciente teve seu quadro agravado por vários fatores: tempo decorrido entre o diagnóstico e a primeira intervenção, que foi de três meses e 10 dias, não retorno do animal conforme solicitado e comportamento extremamente ativo do animal. Também não foi possível o emprego de pinos de maior calibre, por se tratar de um cão da raça Pinscher de $1,5 \mathrm{~kg}$. Esses fatores colaboraram para o insucesso do tratamento, confirmando as observações feitas por Gorse (1998), Harari et al. (1998) e Johnson e DeCamp (1999) em estudos anteriores. Nos outros dois casos não houve a consolidação como esperado, sendo necessário o uso de outro método de fixação. 
Um paciente $(3,5 \%)$ apresentou fratura recorrente, cinco $(17,1 \%)$ apresentaram evolução satisfatória, sem complicações. Cinco $(17,1 \%)$ animais só tinham crostas, houve um $(3,5 \%)$ caso de apoio insatisfatório, que melhorou após retirada do aparelho e instituição de fisioterapia, retornando ao uso satisfatório do membro em uma semana, uma $(3,5 \%)$ fratura de pino, uma $(3,5 \%)$ fratura do acrílico. Um $(3,5 \%)$ animal não retornou para acompanhamento do caso (Fig. 2).

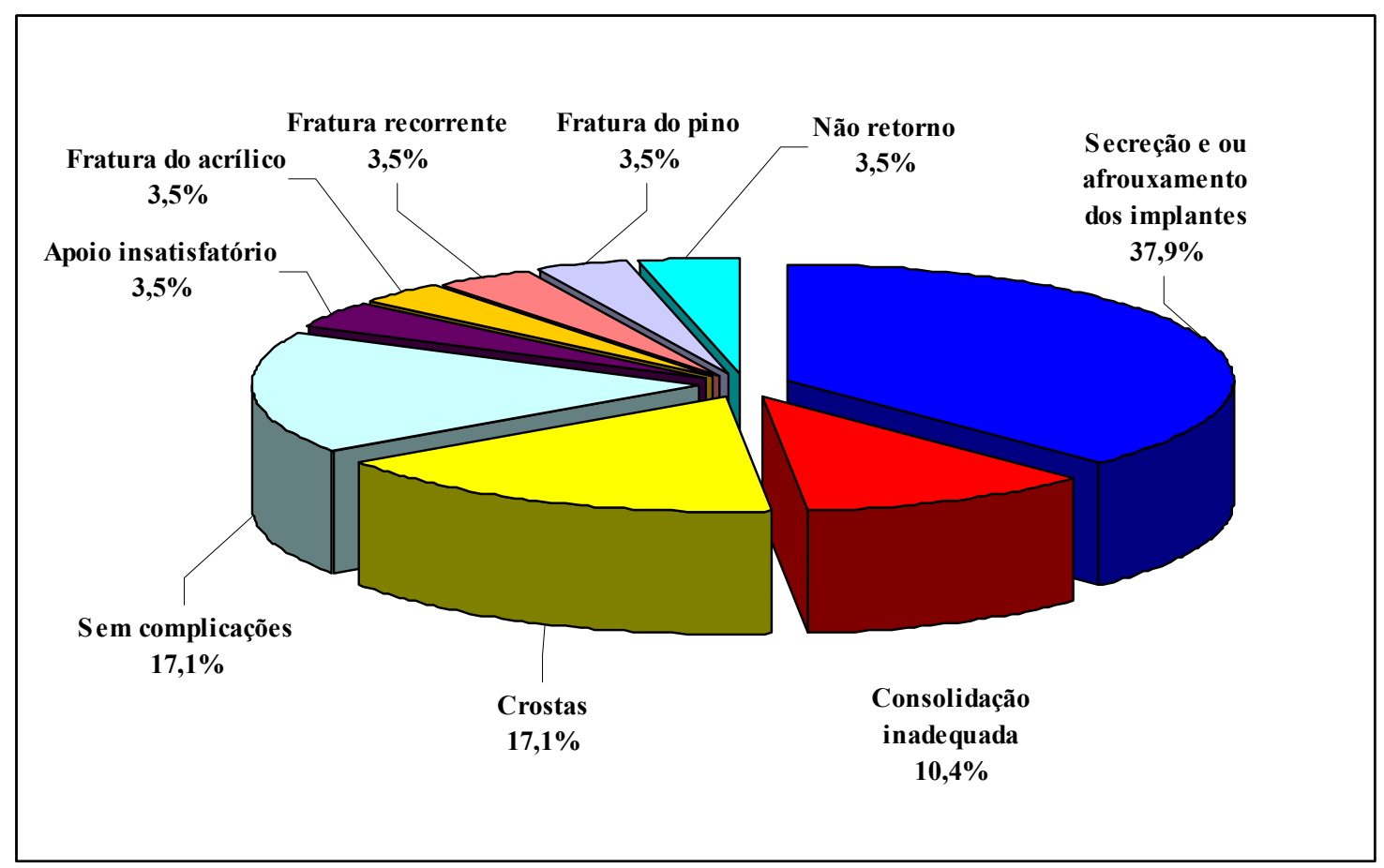

Figura 2. Ocorrência de complicações associadas ao uso da fixação externa em cães tratados no hospital veterinário - EV-UFMG

A fratura recorrente ocorreu dois dias depois da retirada do transfixador após o cão descer de um sofá, ocorrência anteriormente citada por Sisk (1983). O estresse reduzido no córtex ósseo durante o período de recuperação resulta na formação de osso pelo menos parcialmente esponjoso, podendo ocorrer nova fratura, pois o córtex ainda não tem resistência suficiente para suportar as tensões (Piermattei e Flo, 1999).

Um dos casos de fratura exposta apresentou fratura de pino na interface osso-pino, além de ter dois dos quatro restantes frouxos, o que indicou a retirada do aparelho aos 39 dias, período menor do que o esperado, não havendo, entretanto, complicações adicionais. A consolidação se processou como esperado.

O tempo médio de retirada dos aparelhos utilizados no tratamento de fraturas expostas (69 dias) foi muito próximo daquele dos aparelhos usados na consolidação de fraturas limpas $(66,1$ dias), ratificando os bons resultados desta terapêutica no tratamento de fraturas com exposições ósseas. O resultado obtido com as osteotomias corretivas foi satisfatório apesar das complicações como secreção, crostas e, em um caso, fratura do pino, já citadas por Johnson (1992), Fox et al. (1995) e Rezende et al. (2000)

Admite-se que os animais que não retornaram ao hospital tenham ido a alguma outra clínica para retirada do aparelho, não sendo, portanto, colhidos dados de acompanhamento e informações sobre o pós-operatório e suas complicações. O estudo permitiu concluir que secreção e afrouxamento dos pinos são complicações freqüentes neste tipo de fixação, não impedem a consolidação óssea e, na maioria das vezes, está diretamente relacionada com o 
não comparecimento do proprietário com o cão nos retornos programados e não cumprimento das recomendações médicas no pós operatório. A fixação percutânea é aplicável e efetiva no tratamento de fraturas fechadas, de deformidades angulares, bem como no tratamento de fraturas expostas.

Palavras-chave: cão, fixação percutânea, fratura

\section{ABSTRACT}

In this retrospective study 29 dogs of both sexes of several breeds, were evaluated. Complications of external skeletal fixation were common. The most frequent post-surgical complications were chronic pin track drainage or pin loosing $(11 ; 37.9 \%)$, followed by dry crust at the skin-to-pin interface $(5 ; 17.1 \%)$. In five cases (17.1\%) occurred the healing of the fracture without complications. In three dogs $(10.4 \%)$ occurred nonunion and it was used another fixation method. It was observed individual cases of poor limb use (3.5\%) or breakage of the pin (3.5\%) or of the acrylic column (3.5\%). Pin track drainage or loosing was related to the owners care. The most did not come back to the hospital as was demanded or did not follow the instructions to inspect the apparatus daily. This behaviour contributed to increase the rate of post-surgical complications. The external fixation has the advantage to be less invasive and can be used to treat closed and open fractures, limb deformities, nonunion and infected fractures.

Keywords: dog, external fixation, fracture

\section{REFERÊNCIAS BIBLIOGRÁFICAS}

CARNEIRO, L.P. Fixação esquelética externa em cães: Controle pós-operatório (estudo experimental). 2000. 46f. Dissertação (Mestrado em Medicina Veterinária) Universidade Federal de Minas Gerais, Belo Horizonte.

DUDLEY, M.; JOHNSON, A.L.; OLMSTEAD, M. et al. Open reduction and bone plate stabilization, compared with closed reduction and external fixation, for treatment of comminuted tibial fractures: 47 cases (1980-1995) in dogs. $J$. Am. Vet. Med. Assoc., v.211, p.1008-1012, 1997.

EGGER, E.L. Complications of external fixation: a problemoriented approach. Vet. Clin. North Am: Small Anim. Pract., v.21, 1991. p.705-733.

EGGER, E.L. Fraturas de rádio e ulna. In: SLATTER, D. Manual de cirurgia dos pequenos animais. 2.ed. São Paulo: Manole, 1998. v.2, cap.123/132, p.1944-1966/2057-2079.

FOX, S.M.; BRAY, J.C.; GUERIN, S.R. et al. Antebrachial deformities in the dog: treatment with external fixation.$J$. Small Anim. Pract., v.36, p.315-320, 1995.

GORSE, M.J. Using external skeletal fixation for fractures of the radius and ulna and tibia. Vet. Med., v.93, p.463-467, 1998.

HARARI, J. Complications of external skeletal fixation. Vet. Clin. North Am: Small Anim. Pract., v.93. p.445-453, 1992.

HARARI, J.; SEGUIN, B.; PADGETT, S.L. Principles of external skeletal fixation in small-animal sugery. Vet. Med., v.93 .p.445-453, 1998 .
JOHNSON, A.L. Treatment of growth deformities with external skeletal fixation. Vet. Clin. North Am.: Small Anim. Pract., v.22, p.209-223, 1992.

JOHNSON, A.L.; DeCAMP, C.E. External skeletal fixation: linear fixators. Vet. Clin. North Am.: Small Anim. Pract., v.29, p.1135-1152, 1999.

PIERMATTEI, D.L.; FLO, G.L. Manual de ortopedia $e$ tratamento das fraturas dos pequenos animais. 3.ed. São Paulo: Manole, 1999. 694 p.

REZENDE, C.M.F.; MELO, E.G.; LAMAS, M.C.S. et al. Deformidade angular do rádio e da ulna tratada pela distração osteogênica percutânea dinâmica - Relato de caso. Arq. Bras. Med. Vet. Zootec., v.52, p.325-330, 2000.

ROUSH, J.K. Fractures of the tibia. Vet. Clin. North Am.: Small Anim. Pract., v.22, p.161-170, 1992.

RUDD, R.G.; WHITEWHAIR, J. G. Fractures of the radius and ulna. Vet. Clin. North Am.: Small Anim. Pract., v.22, p.135-148, 1992.

SEGUIN, T.D.; HARARI, J.; WOOD, R.D. et al. Bone fracture and sequestration as complications of external skeletal fixation. J. Small Am. Pract., v.38, p. 81-84, 1997.

SHAMIR, M.H.; SHARAR, R.; JOHNSTON, D. E. Semirigid external fixation for repair of fractures in young animals. J. Am. Anim. Hosp. Assoc., v.32, p.521-526, 1996.

SISK, T.D. External fixation. Clin. Orthop. Rel. Res., v.180, p.15-22, 1983. 